\title{
Exploration of the Application of Virtual Reality Technology on Sports Simulation Training
}

\author{
Guangtian Qian ${ }^{1, a}$ \\ ${ }^{1}$ College of Physical Education, Honghe University, Mengzi, Yunnan, China \\ aqianguangtian@163.com
}

Keywords: Sports, Virtual reality technology, Simulation training.

\begin{abstract}
Through the concept of virtual reality technology, the introduction of features, leads in particular application of virtual reality technology in all areas of sports fields; then, from the build simulation training field, a single person special training, remote training with the environment, the core team personnel training, strengthening spot with shortened sports Yucai and other aspects of life expounded the role of virtual reality simulation training in competitive sports in a comprehensive analysis; Finally, we discuss the prospect of virtual reality technology in sports training, thus development of virtual reality technology for China's sports training to provide a reference.
\end{abstract}

\section{Introduction}

For a long time, the world of competitive sports for more and more attention, put a lot of people, financial, material and technical strength, continue to research and explore new sports training theories and methods, in order to improve athletic performance. Especially in Europe and other sports power, we have been committed to high-tech into all aspects of the sports fields. As a born less than 30 years of virtual reality technology also continue to affect the development of competitive sports. China as a sporting nation, want to promote and maintain the stable development of competitive sports, reversing part of sports in front of the world's embarrassing exit, and then transition into the ranks of sports power, but also need the help of high-tech development. In this context it is necessary to focus on virtual reality technology to sports development aid. Based on this, it is to explore the application of virtual technology in this field to make people sad football.

\section{Virtual reality technology}

Virtual reality technology, the image has been called the "spiritual environment", is the use of three-dimensional computer technology to simulate the real environment generated in the space, and by allowing the user a variety of sensing devices "immersion" into the virtual environment, to achieve the user and environmental technology directly interact. As a rising star, VR gradually is superiority and scalability. The virtual reality technology is introduced into the sports field utilization, so that exercise training reflects a more scientific, systematic, innovation. BurdeaG and PhilippeCoiffet made the famous "3I" Theory in 1993 annual meeting of the world's electronics, that VR technology has three salient features: immersion (Immersion), interactivity (Interactivity) and imagination [2]. And these three features with many aspects of sports training and competitions at both fit.

a. Immersion: the essence of VR technology is immersed mainly refers to the user feel completely immersed in the simulated environment. As an advanced virtual reality interactive system, is about human interface technology, giving it generates a variety of sensory stimulating environment, let the users to have a "virtual reality" feeling, you must first meet $94 \%$ of visual and auditory [2]. Scream as a simulated environment in football training, through 3D simulation training field scene graph and image library, including background, geography, weather, sports equipment and related personnel, as well as a variety of sound simulation formal arena audio library, such as the audience sound, the sound of the referee whistle, athletes voices, wind, etc., so as to create a realistic arena athlete environment in which to master pre-configured simulation training standards programs to improve sports skills. 
b. Interactivity: refers to the degree of user control carried out in a simulated environment and receive feedback from simulation training degree of synchronization. Produce such human-computer interaction, mainly by means of various specialized 3D interactive device, as in football simulation training, athletes wearing light clothes data, using stereoscopic glasses, the operation sensing gloves, athletes assigned various virtual equipment operation instruction, and these devices can make real-time feedback response [3]. Natural man - machine and human - human interaction is a distinctive feature of the VR system, so you can "exchange" user with virtual systems.

c. Imagine: the VR system reflects the scalability, virtual environment by human imagination constructed by means of a computer, while people immersed in this virtual environment for learning, training, testing, access to knowledge, tactics, effectively improve exercise capacity [3]. In football simulation training, through simulation and virtual training environment sensing equipment and operations, improve the stadium environment, the ball and the opponent's awareness and proficiency to obtain a successful experience, lessons learned from the simulation training, and to improve the actual combat skills.

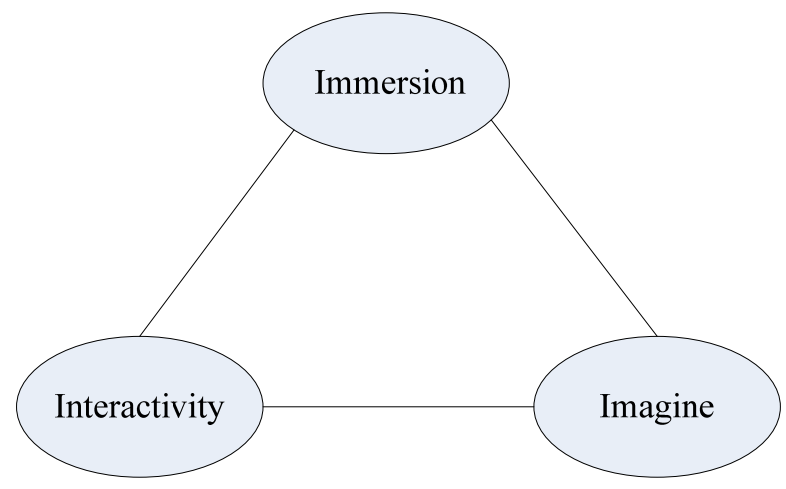

Fig.1 Virtual reality technology

\section{Virtual Reality Technology in Sports Training}

The development of new equipment and systems training and competition, building virtual training scenarios. 1996, developed by the inventor JimRodnunsky skis and snowboards emulator to simulate the famous Colorado ski areas, so do not have to experience the real mountain skiing, you can experience the shock, swinging, rolling, pitching and yawing, etc. real skiing experience. China is one of VR research made sailing training system, which worked on a variety of sensors collect field data, through the operation of a timely start three-dimensional mathematical model of visual construct a realistic virtual training environment, both in this environment no training to meet the conditions (such as the condition of no wind, no waves) to complete the training mission under evaluation and analysis can be performed for a particular athlete [3].

Sports training information collection and analysis, scientific monitoring of sports training.Sports and researchers SheffieldHallam University implements a virtual gymnast system, which by the movement of the real gymnast information collection, analysis, model building, so that the virtual gymnast gymnast gymnastics reproduce precise movements, helping gymnasts improved technical movements, improve the technical level [3]. Tsinghua University and Beijing isomorphic high-tech Co., Ltd. developed a high-tech indoor shooting simulation training systems, the use of sound, light, electricity, sound, high-precision computer virtual reality and other advanced technology to create the environment beyond the reality of the game, focusing on culture trainees observe, analyze, judge and adaptability. Shooters, such as immersive, computer coach will accurately report the number of rings to attack the fire, holding the gun correctness, shooting accuracy and so on. Meanwhile, the system time and hit the percussion parts recorded to the internal memory of the computer quickly, reaching for the training monitoring, storage, training records can easily be commenting on the shooting performance make the determination and statistics.

In the application of high level sports teams. With the rapid development of sports technology, sports events confrontational and more intense competition, technology has become increasingly difficult. Using virtual reality technology, physiological anatomy to follow the laws of human motion, 
combined with the actual situation of the various sports and other virtual operation conducted experiments athletes can improve athletic performance, reduce sports injuries athletes do difficult and complex technical action caused athletes in a virtual lab environment, you can relax and concentrate on doing a variety of exercises, a better grasp of technical movements [4].

\section{Necessity of virtual reality technology implemented in sports}

With the rapid development of sports technology, sports events confrontational and more intense competition, technology has become increasingly difficult. Using virtual reality technology, physiological anatomy to follow the laws of human motion, combined with the actual situation of the various sports and other virtual operation conducted experiments athletes can improve athletic performance, reduce sports injuries athletes do difficult and complex technical action caused athletes in a virtual lab environment, you can relax and concentrate on doing a variety of exercises, a better grasp of technical movements [5], it is necessity (shown in fig2).

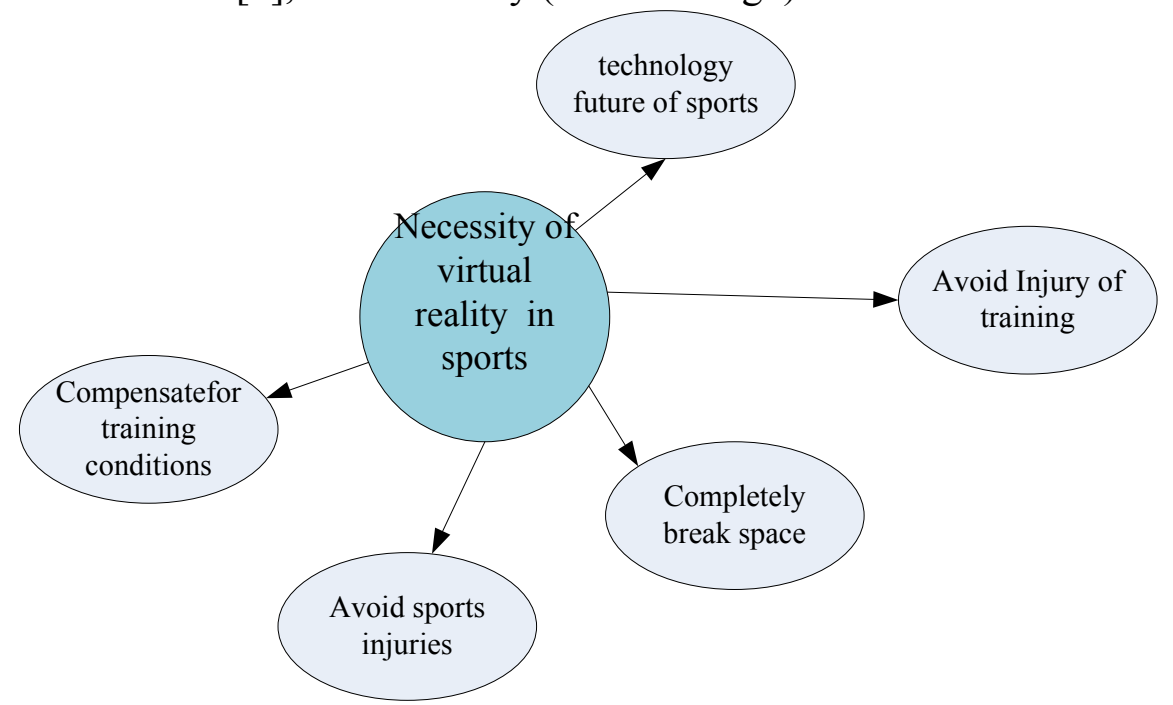

Fig.2 Necessity of virtual reality technology implemented in sports

Compensate for the lack of modern teaching and training conditions.In teaching and training, often because of weather, space, equipment, funding and other aspects, so that some teaching and training sessions impossible [3]. Using virtual reality technology system, you can make up for deficiencies in these areas, students can better understanding of tactics, to get the real feel the same experience, to enrich perceptions and deepen understanding of the content of the teaching.

Avoid difficult and complex technical action brought by sports injuries. With the height of the development of modern technology, confrontational and technical difficulty movement is also growing [2]. Using virtual reality technology virtual experiments can be exempted from this action concerns. Participants in a virtual lab environment, you can safely do all kinds of difficult movements. For example, the altitude of difficult dunks, air tumbling action, high-tech and other action to pull the ball.

Completely break space constraints, time.Using virtual reality technology, you can completely break time and space constraints, we can stay at home to watch the world's technical and tactical and technical guidance of elite athletes and coaches and experience the world-famous modern sports concept. And trains with the world's top athletes, coaches enjoy excellent treatment [5].

Avoid physical education and training of injury.For boxing, taekwondo and other highly confrontational student favorite sports, in teaching is difficult to avoid the occurrence of injuries, many schools simply do not offer courses. Using virtual reality technology virtual practice is exempt from such concerns [6]. Students in a virtual reality environment, you can safely go and fight virtual opponents, but do not worry about any danger. At the same time it will make the evaluation of the student's actions, pointing out deficiencies, improve training efficiency. 
Virtual reality technology - the future of sports science technology.Virtual reality technology in the field of sports, sports will cause a revolution, it will make the sport from a single type to comprehensive, so sports training from experience-based integrated high-tech training to make sports from monotonic trend entertainment, universal type, so that a single physical exercise to exercise therapy [6].

Virtual reality technology applications enable new sports, sports entertainment, the advent of open competition system of virtual reality technology, will create a wide range of sports realm humans give birth to many to send virtual technology as the main Olympic sports The new competition system and sports entertainment; sports training reduces the risk of competition (such as alpine skiing, racing, boarding sport if done intensive training or competition adaptive indoors, it will not appear due to the avalanche, caused by rollovers, etc [7]. The casualties, so athletes in the growing and before the game, reducing injuries during race training); the creation of objective factors cannot reach the realm, in order to make adaptation for athletes training for the object of the game, designed to overcome the opponent's tactics come, the object of an adversary as regular training, will undoubtedly increase the success rate of athletes.

In summary, the important role of virtual reality technology in sport, as well as their application in a variety of sports, so sports field there is a major change, which has important implications for the future of sports and sports unfolds, at the same time, is further evidence that the use of virtual reality technology in sport is essential.

\section{Conclusion}

Although virtual reality technology has an important role in teaching and training, but in the current conditions, there are still many problems waiting to be solved: Virtual reality technology is not mature, with certain limitations, the degree of interaction for further development; currently, most virtual reality devices are manufactured by foreign countries, and the price is quite expensive. Therefore, we need to customize the hardware and software resources to develop virtual reality; virtual reality technology to enhance teachers' understanding of the use. Although the virtual reality technology in the application there are many problems and a high degree of difficulty, but the use of virtual reality technology to further social development is an inevitable trend.

\section{References}

[1] Q.T. Zeng, Discussion about virtual reality "immersion" problem, 'Fujian Radio and TV University, 2009, pp. 15-17.

[2] D.Sh. Han, Variation of the current study formation of world football matches, Shanxi Agricultural University, 2008, pp.06-15.

[3] Q.Ch. Yu and D.Sh Quan,"Virtual Reality Technology in Sports Teaching". Anhui Sports Science, 2005,pp.38-42.

[4] M.G. Yun and X.P. Li , Physical science future new technology --- virtual reality technology, China Sport Science and Technology, 2005, pp73-77.

[5] K.He, Virtual reality technology teaching assistant basketball discussion, Sports Adult Education, 2004,pp.26-29.

[6] Zh.Ch. Feng, Design and implementation of integrated reality technology glide shot put courseware virtual, Wuhan Institute of Physical Education, 2007,pp.11-16.

[7] J. Chen, Virtual reality simulation technology in sport technology application, Sports Science, 2006.pp.82-84. 